\title{
Simultaneous quantification of multiple bacterial metabolites using surface-enhanced Raman scattering
}

Morelli, Lidia; Centorbi, Francesca Alessandra ; llchenko, Oleksii; Jendresen, Christian Bille; Demarchi, Danilo; Nielsen, Alex Toftgaard; Zor, Kinga; Boisen, Anja

\section{Published in:}

Analyst

Link to article, DOI:

10.1039/C8AN02128G

Publication date:

2019

Document Version

Peer reviewed version

Link back to DTU Orbit

Citation (APA):

Morelli, L., Centorbi, F. A., Ilchenko, O., Jendresen, C. B., Demarchi, D., Nielsen, A. T., Zor, K., \& Boisen, A. (2019). Simultaneous quantification of multiple bacterial metabolites using surface-enhanced Raman scattering. Analyst, 144(5), 1600-1607. https://doi.org/10.1039/C8AN02128G

\section{General rights}

Copyright and moral rights for the publications made accessible in the public portal are retained by the authors and/or other copyright owners and it is a condition of accessing publications that users recognise and abide by the legal requirements associated with these rights.

- Users may download and print one copy of any publication from the public portal for the purpose of private study or research.

- You may not further distribute the material or use it for any profit-making activity or commercial gain

- You may freely distribute the URL identifying the publication in the public portal 


\section{Journal name}

\section{ARTICLE}

\section{Simultaneous quantification of multiple bacterial metabolites using surface-enhanced Raman scattering}

Received 00th January 20xx, Accepted 00th January 20xx

DOI: $10.1039 / \times 0 \times x 00000 x$

www.rsc.org/
Lidia Morelli*, Francesca Alessandra Centorbi ${ }^{b}$, Oleksii Ilchenko $^{a}$, Christian Bille Jendresen $^{c}$, Danilo Demarchib, Alex Toftgaard Nielsenc, Kinga Zór ${ }^{\mathrm{a}}$, Anja Boisen ${ }^{\mathrm{a}}$

Given the commercial importance of the compounds produced by genetically modified organisms, there is a need for screening methods which facilitate the evaluation of newly developed strains, especially during the phase of proof-ofconcept development. We report a time-efficient analysis method for the screening of bacterial strains, which enables the detection of two structurally similar secondary bacterial metabolites. By combining liquid-liquid extraction and surfaceenhanced Raman scattering we were able to quantify $p$-coumaric acid and cinnamic acid, produced by genetically modified E. coli from tyrosine and phenylalanine, respectively. With the simple sample pre-treatment method, and by applying a partial least squares data analysis method, we simultaneously detected the analytes from four $E$. coli strains cultured in the presence or absence of tyrosine and phenylalanine.

\section{Introduction}

Metabolic engineering enables the direct modulation of cellular activities by manipulation of enzymatic, transport and regulatory functions, for metabolite overproduction or improvement of cellular properties. ${ }^{1}$ This field gained importance for production of food additives, biofuels and pharmaceuticals, focusing both on the optimization of existing processes and on the development of new ones. ${ }^{2}$ The use of genetically modified organisms is particularly convenient for the production of compounds which are too complex to be chemically synthetized, or too time- and resource-consuming to be extracted from natural sources. ${ }^{3}$ Additionally, although in most cases the production is focused on a single molecule, one organism can be modified to synthetize more than one analyte simultaneously. 4,5

When developing a proof-of-concept microbial strain, several genetic modifications are needed to boost the desired process and prevent the competing ones, due to the complexity of microbial metabolism. ${ }^{6}$ Therefore, a large number of strains must be screened to identify the best performing ones, with repeated optimization cycles resulting in a costly and timeconsuming process, lasting up to several years. ${ }^{2}$ Bioinformatic tools and new technologies have contributed to fast design

\footnotetext{
a. Department of Micro- and Nanotechnology, Technical University of Denmark, 2800 Kgs. Lyngby, DENMARK.

b. Department of Electronics and Telecommunications, Politecnico di Torino, 10129 Torino, ITALY

c. The Novo Nordisk Foundation Center for Biosustainability, Technical University of Denmark, 2800 Kgs. Lyngby, DENMARK.

*e-mail: lidiamorelli90@gmail.com, phone: +4591734340

Electronic Supplementary Information (ESI) available: pure components detected in the standards used for calibration models and spectra reproducibility.
}

and modeling of metabolic pathways, and to rapid genome modification. Nowadays genetic engineering can be performed in massive parallel reactions, resulting in either specific clones or in libraries of different cells. ${ }^{7}$ DNA synthesis for direct use or in gene assembly ${ }^{8,9}$ and techniques such as mutagenesis by UV radiation are also capable of generating myriads of variation. ${ }^{10}$ When constructing genetic manipulations, specific gene insertions can be done in in less than 20 minutes, ${ }^{11}$ however, getting data and selection of the best producing strain is still time consuming. ${ }^{7,12}$ Therefore, there is still a need for fast and high-throughput screening techniques to speed up testing, which is a crucial step and it has been described as the bottleneck of the entire development process. ${ }^{7,12}$

The analytical techniques that are commonly used for analysis (e.g. gas chromatography, high-performance liquid chromatography), are accurate and robust, but they require expensive instrumentation operated by skilled personnel, time-consuming optimization of protocols and long separation procedures. ${ }^{12}$ Raman spectroscopy, a non-destructive, fast and label-free technique, is suitable for collecting molecule-specific information from samples, even in small quantities. ${ }^{13}$ In spite of the advantages offered by Raman spectroscopy, detection of molecules with a small Raman cross-section or at low concentrations in complex media is often challenging. To be able to detect in the aforementioned conditions, high laser power and long acquisition time are required, which could results in the degradation of the analyzed molecules. Additionally, fluorescence can interfere with the Raman signal. ${ }^{14}$ On the other hand, surface-enhanced Raman scattering (SERS) enables detection at low concentration, ${ }^{15}$ or even of single molecules, ${ }^{16}$ due to its ability to enhance the Raman signal of a molecule with reported enhancement factors up to $10^{14}$, through the use of metallic nanostructured 
surfaces. ${ }^{17}$ SERS signal can be collected with a lower laser power and shorter acquisition time than Raman, avoiding biomolecule degradation; ${ }^{18}$ additionally, high signal intensity can overcome fluorescence in biological samples. ${ }^{19}$

Given the advantages and the potential of SERS, this method is being widely explored for a variety of applications in life sciences ${ }^{20-22}$ and microbiology, including identification of pathogen $s^{23-25}$ and metabolomics profiling of bacterial supernatant. ${ }^{26}$ However, one of the limitations of SERS is related to the non-uniformity and instability of SERS substrates, which leads to irreproducible measurements and challenges in the quantification process. As a solution for uniformity across a large surface, Schmidt et al. ${ }^{27}$ developed a silicon metal-capped nanopillar substrate, uniform at a wafer scale, used for detection of low concentrations of hazardous 28,29 and forensic molecules. ${ }^{30}$

In spite of the advantages of SERS-based sensing, the presence of interfering compounds and salts represents another issue for quantitative analysis in complex sample matrices, such as bacterial supernatant. ${ }^{31}$ To address this issue, we have successfully combined SERS-based sensing with sample pretreatment techniques, such as liquid-liquid extraction (LLE) ${ }^{32,33}$ and supported liquid membrane extraction. ${ }^{34}$ Sample pretreatment enabled the separation of the target analyte from interfering compounds in the supernatant matrix. The combination with SERS-based sensing enabled discrimination between different $E$. coli strains based on the amount of a single secondary metabolite in bacterial supernatant. Although quantitative SERS detection is an emerging field, ${ }^{35-38}$ simultaneous quantification of multiple analytes is an additional challenge, often addressed with the use of complex data analysis models. ${ }^{39-43}$

p-Coumaric acid (pHCA) and cinnamic acid (CA) are phenolic compounds with strong antioxidant and antimicrobial activity ${ }^{44}$ used as additives in cosmetics ${ }^{45}$ or food products. ${ }^{46}$ The two analytes, which possess a very similar structure, are produced by genetically modified $E$. coli strains, expressing tyrosine ammonia-lyase (TAL) and/or phenylalanine ammonia-lyase (PAL) with different activities. ${ }^{4}$ Examples were reported of strains producing $\mathrm{pHCA}$ and $\mathrm{CA}$ in the range of $\mathrm{mM} .47,48$ Since mutations to the enzyme may not only alter the activity, but also the substrate specificity, ${ }^{49}$ it is important to measure both products simultaneously. Based on our prior experience, in this work we aimed at quantifying both pHCA and CA produced by four different E.coli strains, cultivated in presence or absence of tyrosine (Tyr) and phenylalanine (Phe). LLE was used as a simple sample pre-treatment step, due to the presence of interfering compounds (e.g. Tyr, Phe and salts). ${ }^{50}$ By using SERS-based detection in combination with LLE as a sample pretreatment method, we significantly reduced the amount of organic solvents needed for the assay and the analysis time per sample. ${ }^{32}$ In addition, a partial least squares (PLS) method was used for quantification and the results were compared with data obtained from HPLC.

\section{Materials and methods}

\section{Chemicals, E. coli cultures and HPLC detection}

Stock solutions of $100 \mathrm{mM}$ pHCA and $100 \mathrm{mM}$ CA were freshly prepared in EtOH 99\%. For SERS calibration standards, pHCA and CA were diluted in dichloromethane (DCM) or in control supernatant (obtained from a non-pHCA/CA producing $E$. coli strain (CBJ786)). In addition, for SERS and HPLC experiments the analytes were diluted in $\mathrm{M} 9$ medium. $\mathrm{HCl} 32 \%$ was used for acidification of samples and DCM as the organic phase for LLE. Stock solutions of $50 \mathrm{mM}$ Phe and $50 \mathrm{mM}$ Tyr were prepared in water and $\mathrm{NaOH}$ at $\mathrm{pH} \sim 14$ respectively.

E. coli strains (CBJ800, CBJ786, CBJ951, CBJ789), expressing TAL and PAL-encoding genes from IPTG-inducible promoters ${ }^{4}$ (Fig. 1), were grown in $\mathrm{M} 9$ medium with $1 \%$ glucose, $2 \mathrm{mM}$ Tyr and/or $2 \mathrm{mM}$ Phe, $1 \mathrm{mM}$ IPTG and antibiotics for maintenance of plasmids for $22 \mathrm{~h}$ as described in our previous work. ${ }^{32}$

For quantification of pHCA and CA produced by E. coli, bacterial supernatant samples were obtained from each strain by centrifugation $\left(10 \mathrm{~min}\right.$ at $10000 \mathrm{~g}, 4{ }^{\circ} \mathrm{C}$ ), and filtration through $0.2 \mu \mathrm{m}$ filters. The concentration of pHCA and CA in samples was measured with reversed-phase HPLC by separation on a HS-F5 column (Sigma-Aldrich, St. Louis, MO, USA) with previously described mobile phases (ammonium formate buffer and acetonitrile), with an overall analysis time of approximately $15 \mathrm{~min}$ per sample. ${ }^{32}$ The absorbance was measured at $333 \mathrm{~nm}$ for pHCA and at $277 \mathrm{~nm}$ for CA. ${ }^{4}$

Aqueous solutions were prepared with ultrapure water obtained from a Milli-Q purification system (Millipore Corporation, Billerica, MA, U.S.), and all the chemicals were purchased from Sigma-Aldrich (St. Louis, MO, USA).

\section{SERS chip fabrication}

The gold-capped silicon nanopillar substrates were fabricated as described by Wu et al., ${ }^{51}$ with 4 min etching time, followed by $1 \mathrm{~min} \mathrm{O}_{2}$ plasma cleaning and deposition of $220 \mathrm{~nm} \mathrm{Au}$ at a rate of $10 \AA / \mathrm{s}$. Wafers were diced with a laser micromachining tool (3D-Micromac AG, D-09126 Chemnitz, Germany) to create $4 \times 4 \mathrm{~mm}^{2}$ chips used for measurements.

\section{SERS data acquisition and analysis}

SERS sensing was performed on $4 \times 4 \mathrm{~mm}^{2}$ substrates using $5 \mu \mathrm{L}$ DCM or $2 \mu \mathrm{L}$ EtOH droplets after the samples were completely dried. The measurements were performed with a DXRxi Raman Imaging Microscope (Thermo Fisher Scientific Inc., Waltham, MA, US). The optical microscope was coupled to a spectrometer $5 \mathrm{~cm}^{-1}$ FWHM and $\pm 2 \mathrm{~cm}^{-1}$ wavenumber accuracy. SERS spectra were collected as previously described ${ }^{34}$ at $780 \mathrm{~nm}$ wavelength with a laser power of $1 \mathrm{~mW}$, 10x objective lens, $50 \mu \mathrm{m}$ slit and $3.6 \mu \mathrm{m}$ diameter estimated laser spot. Maps of 48 points with a $100 \mu \mathrm{m}$ collection step were collected on the surface of each chip, and the spectrum collected in each point was averaged over 3 acquisitions of $0.05 \mathrm{~s}$ each, resulting in a total detection time of approximately $3 \mathrm{~min}$ per sample. 
Data pre-processing and quantitative analysis were performed with MatLab (version 8.4, MathWorks, Natick, MA, US) and TQ Analyst (version 9.2, Thermo Fisher Scientific Inc., Waltham, MA, US). An average SERS spectrum was obtained for each map after polynomial baseline correction ( $7^{\text {th }}$ order) and outlier removal, followed by the application of a partial least squares (PLS) algorithm to build a quantitative model. Standards with known concentrations were used to build the calibration models, and validation samples were randomly chosen for each concentration within the dataset in each case. The models were developed based on a first derivative algorithm in the wavenumber range $800-1700 \mathrm{~cm}^{-1}$, in conjunction with quadratic baseline removal.

\section{Sample extraction}

The experimental procedure for LLE, based on Krygier et $a .^{52}$ is presented in Fig. 2. The extraction was carried out as previously described by Morelli et al. ${ }^{32}$ using $500 \mu \mathrm{L}$ of bacterial supernatant or spiked M9 medium containing PHCA, $\mathrm{CA}$ and/or Tyr and Phe. The sample was acidified with $50 \mu \mathrm{L}$ $32 \% \mathrm{HCl}$ (aq) (Fig. 2a) and vortexed for optimal mixing. $500 \mu \mathrm{L}$ DCM was added to the sample (Fig. $2 b$ ) and vortexed again, followed by $30 \mathrm{~min}$ static incubation at room temperature. The aqueous phase was then removed and diluted 10 times with phosphate buffer ( $\mathrm{pH} \mathrm{8,0.04} \mathrm{M)} \mathrm{for} \mathrm{HPLC} \mathrm{analysis} \mathrm{(Fig.} \mathrm{2c),} \mathrm{in}$ order to increase the $\mathrm{pH}$ to 6 , whereas the organic phase was directly used for SERS analysis (Fig. $2 \mathrm{~d}$ and e).

\section{Results and discussion}

\section{LLE and SERS for extraction and detection of pHCA and CA}

As described in our previous works, ${ }^{31,32,34}$ genetically modified E. coli strains are cultured in a growth medium containing appropriate salts, nutrients and antibiotics, with substrates (e.g. Tyr, Phe) at starting concentrations up to $2 \mathrm{mM}$. According to the genes expressed by each strain, and according to their synthesis efficiency, substrates can be found at high concentrations in bacterial supernatant.

The SERS spectra of Tyr and Phe in EtOH, highlighted in red and black respectively in Fig. 3a, partially overlap with the signal of pHCA and CA (purple and blue graphs). For instance, both CA and Phe present a peak at $1000 \mathrm{~cm}^{-1}$, whereas overlapping peaks can be found in the region $1100-1300 \mathrm{~cm}^{-1}$ for Tyr, pHCA and CA. Therefore, all the molecules contribute to the SERS spectrum of the mixture (Fig. 3a, green) to a different extent.

LLE, a common extraction method for phenolic compounds, ${ }^{53-}$ 55 was used for separation of pHCA and CA, according to the experimental procedure depicted in Fig. 2. LLE enables the partition of a compound between two immiscible phases. In general, uncharged compounds tend to be more soluble in organic solvents, whereas charged molecules tend to stay in the aqueous phase. By regulating the $\mathrm{pH}$ of the aqueous sample, uncharged molecules, such as pHCA and CA, can be extracted to the organic phase whereas charged molecules (e.g Tyr, Phe and salts present in the growth medium) remain in the aqueous phase.

In our previous works, we combined LLE and the here exploited gold-capped silicon nanopillar substrates for extraction and SERS detection of pHCA. ${ }^{32,33}$ Besides excluding Tyr and salts, measuring the target analyte in the organic phase (DCM) provided the optimal working conditions for metal-capped nanopillar substrates, since it was demonstrated that wetting the substrate with an organic solvent improves the leaning of silicon nanopillars, ${ }^{56}$ leading to a better SERS signal enhancement.

With LLE we were able to extract both pHCA and CA from aqueous samples. The effective exclusion of Tyr and Phe was demonstrated by performing LLE on medium spiked with Tyr and Phe only (Fig. 3b, light blue). It can be observed that the obtained signal was comparable to the one recorded from the control (Fig. 3b, orange). Additionally, when LLE was not performed, no visible vibrational peaks could be recorded, even after diluting the sample with EtOH (Fig. 3b, brown). ${ }^{32,34}$ In Fig. $3 b$, the green spectrum represents the signal obtained after LLE of a sample containing pHCA, CA, Tyr and Phe. Vibrational peaks originating from both pHCA (e.g. at $1169 \mathrm{~cm}$ ${ }^{1}$ ) and CA (e.g. at $1000 \mathrm{~cm}^{-1}$ ) could be observed, as also shown in Fig. 3a, without the influence of Tyr and Phe. Therefore, LLE enabled the exclusion of both salts and substrates with interfering vibrational peaks.

\section{Quantification of pHCA and CA spiked in M9 medium}

As previously described, LLE enables the partition of a compound between two immiscible liquid phases, according to the affinity of the compound towards each phase. The partition of pHCA and CA in this work was evaluated through the extraction efficiency $(\mathrm{EE})$, calculated as:

$$
\% E E=\frac{[S]_{2}}{[S]_{i}} \cdot 100
$$

where $[S]_{i}$ is the initial concentration, known in spiked samples, and $[S]_{2}$ is the concentration of $S$ in the organic phase after extraction.

Based on our previous study, ${ }^{32}$ we found that EE was $9.8 \pm 1.1$ $\%$ for pHCA. However, in the case of simultaneous detection of pHCA and CA, it was important to evaluate the EE of CA as well as of both analytes in solution.

Samples of M9 medium were used, spiked with $500 \mu \mathrm{M}$ of pHCA and CA in different proportions (pHCA/CA: 500/0, $400 / 100,300 / 200,200 / 300,100 / 400,0 / 500 \mu \mathrm{M})$; the concentration range was similar to the one expected in bacterial supernatant (see Fig. $5 \mathrm{c}$ ). After LLE, the aqueous phase was removed, diluted 10 times with phosphate buffer $(\mathrm{pH} 8)$ and measured with HPLC in order to calculate the concentration of pHCA and CA left in aqueous phase, as depicted in Fig. 2. The EE of pHCA was confirmed to be $9.4 \pm$ 
$1.1 \%$, whereas the EE of CA was found to be $85.9 \pm 1 \%$. CA has one $\mathrm{OH}$ group less than $\mathrm{pHCA}$, possibly leading to a higher affinity of CA towards the organic phase, and therefore to a higher EE. In both cases, when we extracted pHCA and CA in mixture we found the EE was comparable to the one obtained with extraction of single analytes $\left(\mathrm{EE}_{\mathrm{pHCA}} 9.8 \pm 1.1 \%\right.$, $\mathrm{EE}_{\mathrm{CA}} 84.3$ $\pm 0.3 \%)$.

In order to perform quantification of spiked M9 samples with SERS, a PLS calibration model based on spiked DCM samples was constructed with TQ Analyst. The concentration of pHCA and CA in DCM was chosen based on the expected analyte concentration in supernatant (approximately between 0 and $600 \mu \mathrm{M})$ and on the previously calculated EE for both pHCA and $C A$. Due to the different $E E$, the concentration range of pHCA was between 0 and $100 \mu \mathrm{M}$, whereas the concentration range of CA was between 0 and $500 \mu \mathrm{M}$ (pHCA/CA: 100/0, $80 / 125,50 / 250,20 / 375,0 / 500 \mu \mathrm{M})$. The results of the PLS calibration model are shown in Fig. $4 a$ and $b$. The obtained PLS model proved to be suitable for calibration and prediction of pHCA concentration (RMSEC $=5.65 \mu \mathrm{M}, \mathrm{r}^{2}=0.989$ and RMSEP $=2.49 \mu \mathrm{M}, r^{2}=0.998$, LOD $\left.=3 \mu \mathrm{M}\right)$, and of CA (RMSEC $=21.7$ $\mu \mathrm{M}, \mathrm{r}^{2}=0.993$ and RMSEP $=35.6 \mu \mathrm{M}, \mathrm{r}^{2}=0.984, \mathrm{LOD}=50$ $\mu \mathrm{M})$, and the validity of the model was confirmed by the successful separation of pHCA, CA and background pure components (Fig. S1).

The presented calibration model was applied to the spiked M9 samples previously analyzed through HPLC. DCM extracts were measured through SERS, and PHCA and CA were quantified through the calibration model. The data points in Fig. $4 \mathrm{c}$ and $\mathrm{d}$ were calculated by dividing pHCA and CA concentrations obtained from PLS quantification by the respective $E E$, resulting in values close to the spiked concentrations.

\section{Screening of bacterial strains}

In spite of the promising data obtained from analytes spiked in M9 medium, the quantification of PHCA and CA in real supernatant samples was not possible with the presented calibration model (data not shown), due to the higher matrix complexity of supernatant extracts. Previously we observed that other compounds besides pHCA and CA were possibly extracted in the organic phase. ${ }^{32}$ Therefore, in order to overcome the nonspecifically extracted compounds, a different calibration model was implemented, which better mimicked the complexity of real samples. Calibration samples were obtained by extracting pHCA and CA from spiked control supernatant samples, and measuring DCM extracts with SERS. The control supernatant was obtained from $E$. coli strain CBJ786, a non-pHCA/CA producing strain, cultured in the same conditions as the producing ones. Control supernatant samples were spiked with $600 \mu \mathrm{M}$ pHCA and CA in different proportions (pHCA/CA: 600/0, 480/120, 300/300, 120/480, $0 / 600 \mu \mathrm{M}$ ) and processed with LLE. DCM extracts were measured with SERS and used to construct the calibration models shown for pHCA and CA in Fig. $5 a$ and $b$ respectively.
When using the control supernatant as a sample matrix, the model proved to be suitable for calibration and quantification of pHCA (RMSEC $=46.8 \mu \mathrm{M}, r^{2}=0.977$ and RMSEP $=40.9 \mu \mathrm{M}$, $r^{2}=0.993$, LOD $\left.=90 \mu \mathrm{M}\right)$, and CA (RMSEC $=28.1 \mu \mathrm{M}, \mathrm{r}^{2}=0.992$ and RMSEP $\left.=29.8 \mu \mathrm{M}, \mathrm{r}^{2}=0.992, \mathrm{LOD}=70 \mu \mathrm{M}\right)$.

As shown in Fig. S2, the model was able to separate the signal of the analytes from the background. However, the influence of the background signal recorded from supernatant controls was more significant than in the case of M9 controls, and the analyte spectra had a few differences compared to pHCA and CA SERS spectra in EtOH. For instance, we found a prominent peak originating from the control samples at $1000 \mathrm{~cm}^{-1}$, therefore the overlapping characteristic CA peak could not be used for CA quantification. Nevertheless, significant spectral features were successfully identified for both pHCA and CA, such as the pHCA peak at $1169 \mathrm{~cm}^{-1}$, and the CA peak proportion in the $1580-1660 \mathrm{~cm}^{-1}$ spectral region.

Bacterial supernatant samples were obtained from $E$. coli strains CBJ786, CBJ951, CBJ800 and CBJ789, cultured as described in Materials and Methods. According to the presence of Tyr and/or Phe in the growth medium (indicated in Fig. $5 c$ by $T$ and/or $P$ next to the number of the corresponding strain), different concentrations of pHCA and CA were obtained. Supernatant samples were processed with LLE and the extracts were measured with SERS (Fig. S3), applying the calibration models in Fig. $5 a$ and $b$ for quantification. With this method, as shown in Fig. 5c, pHCA and CA were successfully quantified in real supernatant samples, with results in good agreement with HPLC.

The pre-treatment time was $35 \mathrm{~min}$ for all the samples, plus 3 min for SERS acquisition of each sample, versus $15 \mathrm{~min}$ for each HPLC run, with a significant gain in time-to-result. Moreover, the detected pHCA and CA concentrations were within or below ranges reported in several studies, ${ }^{47,48,57-61}$ proving that the developed method is suitable for differentiating engineered microbial strains overproducing pHCA and CA.

\section{Conclusions}

In this work we demonstrated the simultaneous extraction and quantification of bacterial metabolites in $E$. coli supernatant. The advantages of the previously developed method, based on LLE sample pre-treatment and SERS acquisition, were confirmed and used in combination with a simple PLS model for quantification of multiple analyte mixtures in real supernatant samples. The result was a simple and effective method for fast and quantitative screening of bacterial strains, based on the amount of produced metabolites. The presented work demonstrates the applicability of the method during the development of a proof-of-concept strain, where there is a need for a time- and cost-efficient screening of a large number of strains.

\section{Acknowledgements}


The research was funded by Danish National Research Foundation (DNRF122), Villum Fonden (Grant No. 9301), the European Research Council (Grant No. 320535, 'HERMES'). Christian Bille Jendresen was supported by The Novo Nordisk Foundation (Grant No. NNF15OC0015246).

We also acknowledge Hemanshu Mundhada for help with HPLC analysis.

\section{Conflict of interest}

There are no conflicts of interest to declare.

\section{References}

J. Bailey, Science, 1991, 252, 1668-1675.

J. Nielsen and J. D. Keasling, Cell, 2016, 164, 1185-1197.

P. Saling, Appl. Microbiol. Biotechnol., 2005, 68, 1-8.

C. B. Jendresen, S. G. Stahlhut, M. Li, P. Gaspar, S. Siedler, J. Förster, J. Maury, I. Borodina and A. T. Nielsen, Appl.

Environ. Microbiol., 2015, 81, 4458-4476.

N. Kallscheuer, M. Vogt, A. Stenzel, J. Gätgens, M. Bott and J. Marienhagen, Metab. Eng., 2016, 38, 47-55. Bioeng. Biotechnol., 2015, 3, 1-11. J. K. Rogers and G. M. Church, Trends Biotechnol., 2016, 34 198-206.

M. T. Bonde, S. Kosuri, H. J. Genee, K. Sarup-Lytzen, G. M. Church, M. O. A. Sommer and H. H. Wang, ACS Synth. Biol., 2015, 4, 17-22. G. M. Church, 2014

10 M. T. Reetz, A. Zonta, K. Schimossek, K. Liebeton and K. E. Jaeger, Angew. Chemie (International Ed. English), 1997, 36, 2830-2832.

11 D. G. Gibson, L. Young, R. Y. Chuang, J. C. Venter, C. A. Hutchison and H. O. Smith, Nat. Methods, 2009, 6, 343345.

12 J. A. Dietrich, A. E. McKee and J. D. Keasling, Annu. Rev. Biochem., 2010, 79, 563-590.

13 L. A. Lyon, C. D. Keating, A. P. Fox, B. E. Baker, L. He, S. R. Nicewarner, S. P. Mulvaney and M. J. Natan, Anal. Chem. 1998, 70, 341-362.

14 T. Vankeirsbilck, A. Vercauteren, W. Baeyens, G. Van der Weken, F. Verpoort, G. Vergote and J. . Remon, TrAC Trends Anal. Chem., 2002, 21, 869-877.

15 A. Kim, S. J. Barcelo, R. S. Williams and Z. Li, Anal. Chem., 2012, 84, 9303-9.

16 S. Nie, Science, 1997, 275, 1102-1106.

17 K. Kneipp, H. Kneipp, I. Itzkan, R. R. Dasari and M. S. Feld, Chem. Rev., 1999, 99, 2957-2976.

18 E. Smith and G. Dent, Modern Raman Spectroscopy-A Practical Approach, John Wiley \& Sons, Ltd, 2005, vol. 36. D. Wei, S. Chen and Q. Liu, Appl. Spectrosc. Rev., 2015, 50, 387-406.

20

M. Navas-Moreno, M. Mehrpouyan, T. Chernenko, D. Candas, M. Fan, J. J. Li, M. Yan and J. W. Chan, Sci. Rep.,
2017, 7, 4471.

I-Fang Cheng, H.-C. Chang, T.-Y. Chen, C. Hu and F.-L. Yang, Sci. Rep., 2013, 3, 2365.

R. M. Jarvis, R. Goodacre, P. O. Box, S. Street and M. Manchester, Anal. Chem., 2004, 76, 40-7.

A. März, B. Mönch, P. Rösch, M. Kiehntopf, T. Henkel and J. Popp, Anal. Bioanal. Chem., 2011, 400, 2755-2761. A. Mühlig, T. Bocklitz, I. Labugger, S. Dees, S. Henk, E. Richter, S. Andres, M. Merker, S. Stöckel, K. Weber, D. Cialla-May and J. Popp, Anal. Chem., 2016, 88, 7998-8004. R. K. Lauridsen, T. Rindzevicius, S. Molin, H. K. Johansen, R. W. Berg, T. S. Alstrøm, K. Almdal, F. Larsen, M. S. Schmidt and A. Boisen, Sens. Bio-Sensing Res., 2015, 5, 84-89. W. R. Premasiri, J. C. Lee, A. Sauer-Budge, R. Théberge, C. E. Costello and L. D. Ziegler, Anal. Bioanal. Chem., 2016, 408, 4631-4647.

M. S. Schmidt, J. Hübner and A. Boisen, Adv. Mater., 2012, 24, 11-18.

A. Hakonen, T. Rindzevicius, M. S. Schmidt, P. O. Andersson, L. Juhlin, M. Svedendahl, A. Boisen and M. Käll, Nanoscale, 2016, 8, 1305-1308.

A. Hakonen, F. Wang, P. O. Andersson, H. Wingfors, T. Rindzevicius, M. S. Schmidt, V. R. Soma, S. Xu, Y. Li, A. Boisen and H. Wu, ACS Sensors, 2017, 2, 198-202.

A. Hakonen, K. Wu, M. Stenbæk Schmidt, P. O. Andersson, A. Boisen and T. Rindzevicius, Talanta, 2018, 189, 649-652. L. Morelli, C. B. Jendresen, K. Zor, T. Rindzevicius, M. S. Schmidt, A. T. Nielsen and A. Boisen, Procedia Technol., 2017, 27, 190-192.

L. Morelli, K. Zór, C. B. Jendresen, T. Rindzevicius, M. S. Schmidt, A. T. Nielsen and A. Boisen, Anal. Chem., 2017, 89, 3981-3987.

L. Morelli, L. Serioli, F. A. Centorbi, C. B. Jendresen, M. Matteucci, O. Ilchenko, D. Demarchi, A. T. Nielsen, K. Zór and A. Boisen, Lab Chip, 2018, 18, 869-877.

L. Morelli, S. Z. Andreasen, C. B. Jendresen, A. T. Nielsen, J. Emnéus, K. Zór and A. Boisen, Analyst, 2017, 142, 45534559.

C. Westley, Y. Xu, B. Thilaganathan, A. J. Carnell, N. J. Turner and R. Goodacre, Anal. Chem., 2017, 89, 24722477.

S. Fornasaro, S. D. Marta, M. Rabusin, A. Bonifacio and V. Sergo, Faraday Discuss., 2016, 187, 485-499.

E. Kämmer, K. Olschewski, S. Stöckel, P. Rösch, K. Weber, D. Cialla-May, T. Bocklitz and J. Popp, Anal. Bioanal. Chem., 2015, 407, 8925-8929.

O. Durucan, T. Rindzevicius, M. S. Schmidt, M. Matteucci and A. Boisen, ACS Sensors, 2017.

O. O. Ilchenko, Y. V. Pilgun, A. S. Reynt and A. M. Kutsyk, Ukr. J. Phys., 2016, 61, 519-522.

B. Li, B. H. Ray, K. J. Leister and A. G. Ryder, Anal. Chim. Acta, 2013, 796, 84-91.

C. Cannizzaro, M. Rhiel, I. Marison and U. Von Stockar, Biotechnol. Bioeng., 2003, 83, 668-680.

S. Zou, M. Hou, J. Li, L. Ma and Z. Zhang, Sci. Rep., 2017, 7, 1-9.

43 A. I. Radu, O. Ryabchykov, T. W. Bocklitz, U. Huebner, K. 
Weber, D. Cialla-May and J. Popp, Analyst, 2016, 141, 4447-4455.

44 Y. Kadoma and S. Fujisawa, Molecules, 2008, 13, 24882499.

45 A. Ribeiro, M. Estanqueiro, M. Oliveira and J. Sousa Lobo, Cosmetics, 2015, 2, 48-65.

46 N. Balasundram, K. Sundram and S. Samman, Food Chem., 2006, 99, 191-203.

47 K. Nijkamp, N. van Luijk, J. A. M. de Bont and J. Wery, Appl. Microbiol. Biotechnol., 2005, 69, 170-177.

48 K. Nijkamp, R. G. M. Westerhof, H. Ballerstedt, J. A. M. de Bont and J. Wery, Appl. Microbiol. Biotechnol., 2007, 74, 617-624.

49 A. Nobili, M. G. Gall, I. V. Pavlidis, M. L. Thompson, M. Schmidt and U. T. Bornscheuer, FEBS J., 2013, 280, 30843093.

50 S. Stewart and P. . Fredericks, Spectrochim. Acta Part A Mol. Biomol. Spectrosc., 1999, 55, 1641-1660.

51 K. Wu, T. Rindzevicius, M. S. Schmidt, K. B. Mogensen, A. Hakonen and A. Boisen, J. Phys. Chem. C, 2015, 119, 20532062. K. Krygier, F. Sosulski and L. Hogge, J. Agric. Food Chem., 1982, 30, 330-334.

53 P. Garcia-Salas, A. Morales-Soto, A. Segura-Carretero and A. Fernández-Gutiérrez, Molecules, 2010, 15, 8813-8826.

54 M. Naczk and F. Shahidi, J. Chromatogr. A, 2004, 1054, 95111.

55 N. J. P. Wierckx, H. Ballerstedt, J. a M. De Bont and J. Wery, Appl. Environ. Microbiol., 2005, 71, 8221-8227. A. Kim, S. J. Barcelo, R. S. Williams and Z. Li, Anal. Chem., 2012, 84, 9303-9.

57 T. Vannelli, W. Wei Qi, J. Sweigard, A. A. Gatenby and F. S. Sariaslani, Metab. Eng., 2007, 9, 142-151.

58 Y. Xue, Y. Zhang, D. Cheng, S. Daddy and Q. He, Proc. Natl. Acad. Sci., 2014, 111, 9449-9454.

59 S. Noda, T. Miyazaki, T. Miyoshi, M. Miyake, N. Okai, T. Tanaka, C. Ogino and A. Kondo, J. Ind. Microbiol. Biotechnol., 2011, 38, 643-648. Y. Kawai, S. Noda, C. Ogino, Y. Takeshima, N. Okai, T. Tanaka and A. Kondo, Microb. Cell Fact., 2013, 12, 1-9.

61 A. Rodriguez, K. R. Kildegaard, M. Li, I. Borodina and J. Nielsen, Metab. Eng., 2015, 31, 181-188. 


\section{Figures}

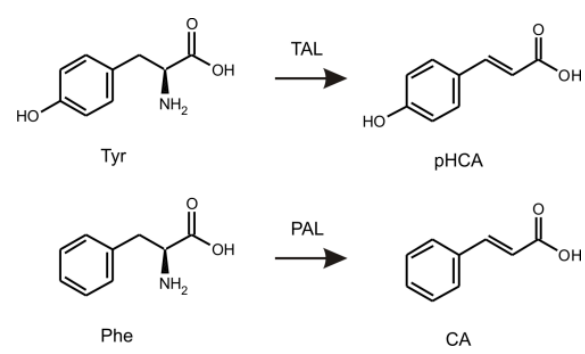

Fig. $1 \mathrm{TAL}$ and PAL enzymatic reactions. 


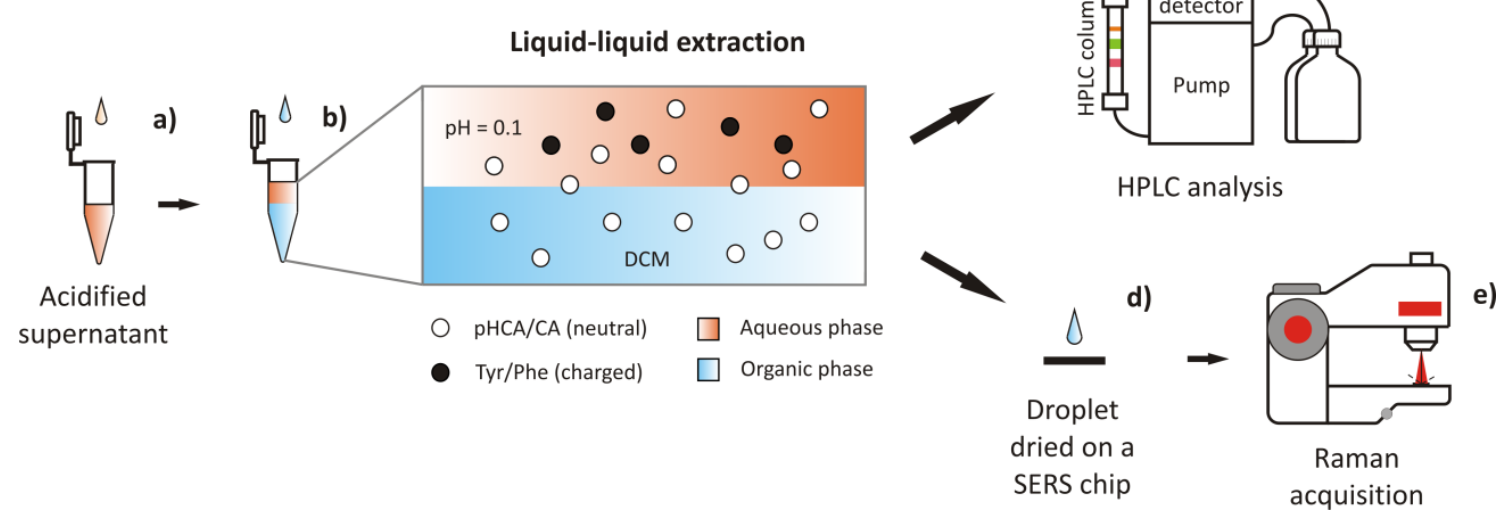

Fig. 2 Experimental procedure for extraction of pHCA/CA from E. coli supernatant or spiked M9 medium samples. (a) The samples were acidified at $\mathrm{pH} 0.1$ and (b) DCM was added to enable liquid-liquid extraction (inset). (c) The concentration of pHCA and CA in aqueous phase after extraction was analyzed with HPLC, whereas (d) droplets of organic phase were poured on SERS substrates, dried and (e) collected with a Raman microscope. 

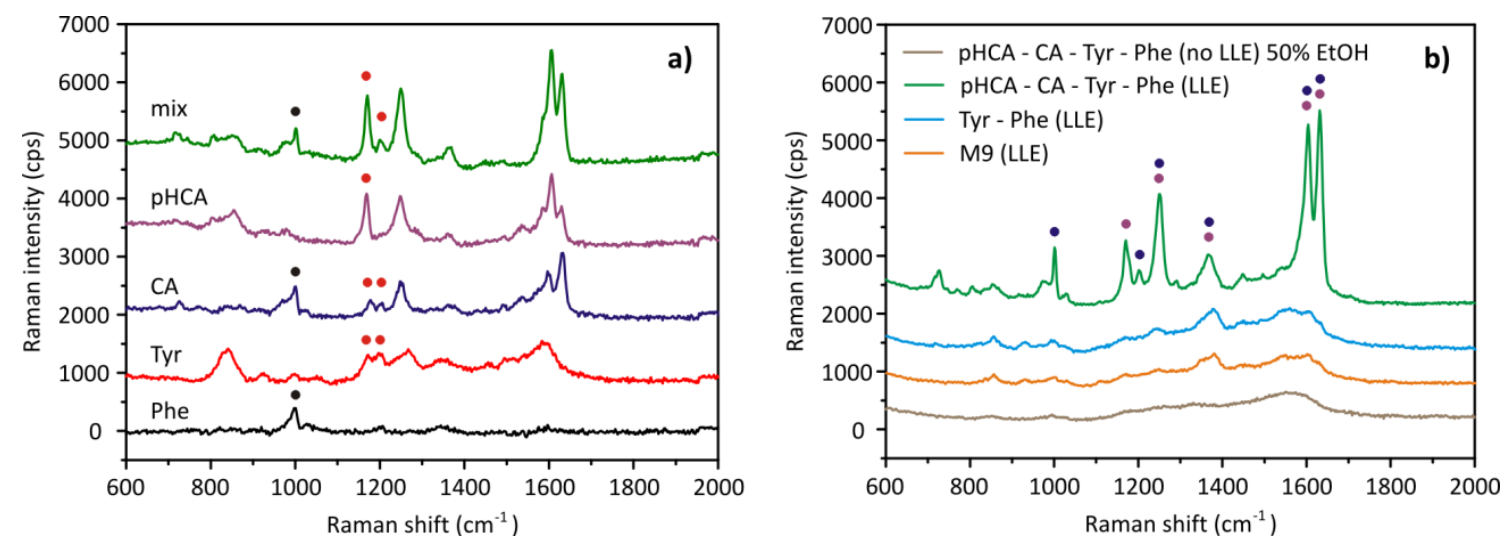

Fig. 3 (a) SERS spectra of $500 \mu \mathrm{M}$ pHCA, CA, Tyr and Phe in EtOH 99\% (green) and of $500 \mu \mathrm{M}$ of each analyte, after background subtraction. Black and red dots highlight the vibrational peaks of Phe and Tyr respectively, which can also be found in CA and pHCA spectra. (b) SERS spectra of M9 medium spiked with $500 \mu \mathrm{M} \mathrm{pHCA}, \mathrm{CA}$, Tyr and Phe 2-fold diluted with EtOH 99\% (brown) prior to liquid-liquid extraction (LLE), and of organic extracts of medium spiked with the 4 analytes (green), with only Tyr and Phe (light blue), and with no analytes (orange). The spectral features of pHCA and CA, overlapped in the mixture, are highlighted with purple and blue dots respectively. The spectra were shifted with arbitrary offsets for ease of representation. 

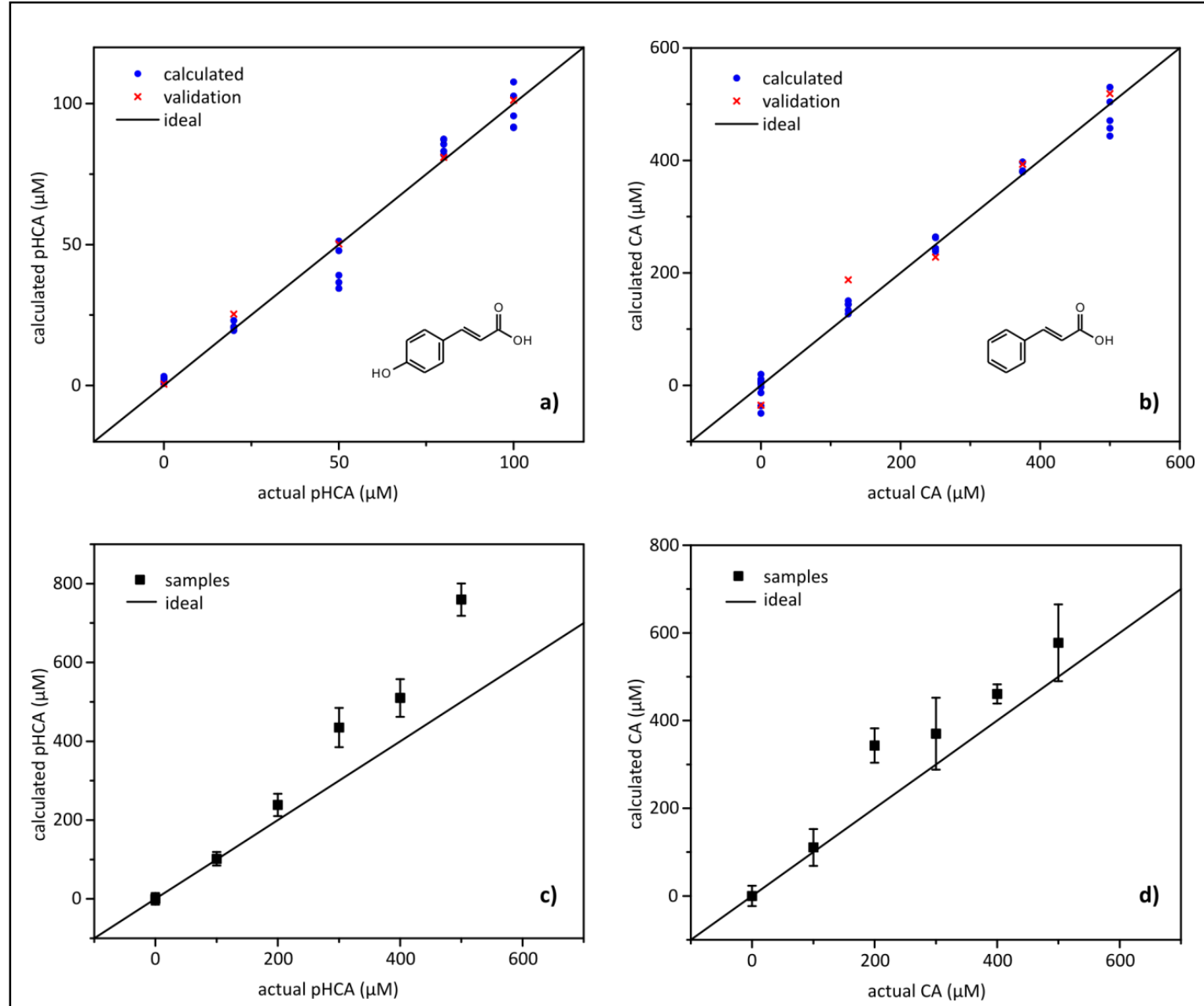

Fig. 4 PLS calibration model in DCM for (a) pHCA and (b) CA, with each point representing a SERS map (data collected on triplicate chips with 2 maps on each chip, $n=6$ ). (c and d) Quantification of the mixture spiked in M9. SERS data were collected on triplicate chips, with two maps on each chip, with error bars representing standard deviation $(n=6)$. 

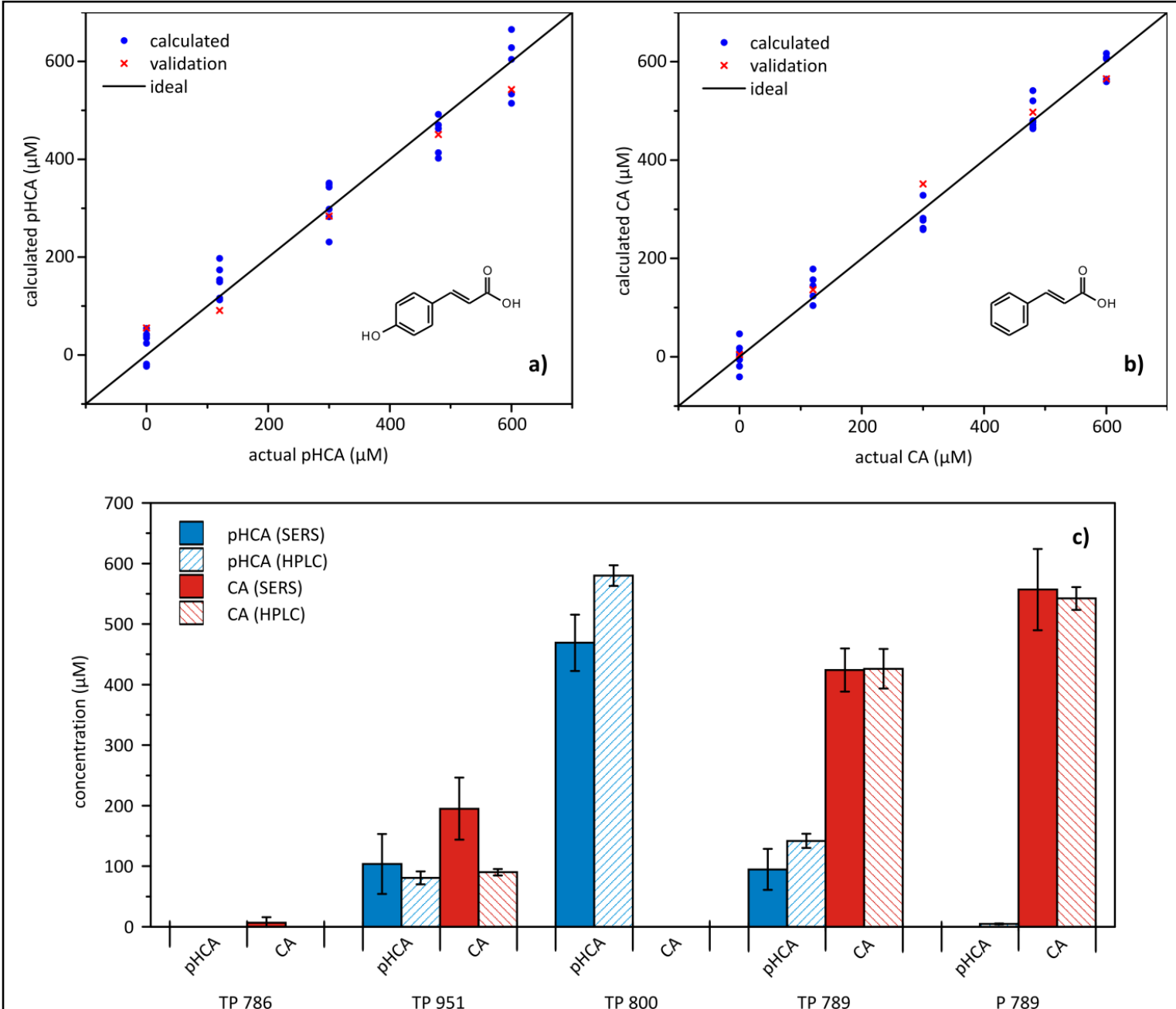

Fig. 5 PLS calibration model of (a) pHCA and (b) CA in spiked supernatant samples pre-treated with LLE, with each point representing a SERS map (data collected on triplicate chips with 2 maps on each chip, $n=6$ ). (c) SERS quantification of real samples compared with HPLC data. T and P indicate Tyr and/or Phe enriched growth medium for E. coli pHCA and CA production. SERS data were collected on triplicate chips, with two maps on each chip $(n=6)$, whereas HPLC data were averaged over triplicate injections $(n=3)$. 